\title{
A Family of Uranyl Coordination Polymers Containing O-donor Dicarboxylates and Trispyridyltriazine (TPTZ) Guests
}

Sonia G. Thangavelu and Christopher L. Cahill ${ }^{*}$

Department of Chemistry, The George Washington University, $80022^{\text {nd }}$ Street, NW, Washington, DC, 20052, USA

* Corresponding author. Phone: (202) 994-6959. Email: cahill@gwu.edu

\section{Supporting Information}

Table S1. Selected bond lengths $(\AA)$ and bond angles $\left(^{\circ}\right)$ for the uranyl centers in $\mathbf{1}$.

\begin{tabular}{|lc|lc|}
\hline $\mathrm{U}(1)-\mathrm{O}(1) \# 1$ & $1.762(3)$ & $\mathrm{O}(2) \# 1-\mathrm{U}(1)-\mathrm{O}(3)$ & $64.21(9)$ \\
$\mathrm{U}(1)-\mathrm{O}(1)$ & $1.762(3)$ & $\mathrm{O}(3) \# 1-\mathrm{U}(1)-\mathrm{O}(3)$ & $180.00(12)$ \\
$\mathrm{U}(1)-\mathrm{O}(2)$ & $2.418(3)$ & $\mathrm{O}(1) \# 1-\mathrm{U}(1)-\mathrm{O}(4)$ & $90.01(12)$ \\
$\mathrm{U}(1)-\mathrm{O}(2) \# 1$ & $2.418(3)$ & $\mathrm{O}(1)-\mathrm{U}(1)-\mathrm{O}(4)$ & $89.99(12)$ \\
$\mathrm{U}(1)-\mathrm{O}(3) \# 1$ & $2.500(3)$ & $\mathrm{O}(2)-\mathrm{U}(1)-\mathrm{O}(4)$ & $64.30(9)$ \\
$\mathrm{U}(1)-\mathrm{O}(3)$ & $2.500(3)$ & $\mathrm{O}(2) \# 1-\mathrm{U}(1)-\mathrm{O}(4)$ & $115.70(9)$ \\
$\mathrm{U}(1)-\mathrm{O}(4)$ & $2.516(3)$ & $\mathrm{O}(3) \# 1-\mathrm{U}(1)-\mathrm{O}(4)$ & $128.41(9)$ \\
$\mathrm{U}(1)-\mathrm{O}(4) \# 1$ & $2.516(3)$ & $\mathrm{O}(3)-\mathrm{U}(1)-\mathrm{O}(4)$ & $51.59(9)$ \\
$\mathrm{O}(1) \# 1-\mathrm{U}(1)-\mathrm{O}(1)$ & 180.0 & $\mathrm{O}(1) \# 1-\mathrm{U}(1)-\mathrm{O}(4) \# 1$ & $89.99(12)$ \\
$\mathrm{O}(1) \# 1-\mathrm{U}(1)-\mathrm{O}(2)$ & $88.42(11)$ & $\mathrm{O}(1)-\mathrm{U}(1)-\mathrm{O}(4) \# 1$ & $90.01(12)$ \\
$\mathrm{O}(1)-\mathrm{U}(1)-\mathrm{O}(2)$ & $91.58(11)$ & $\mathrm{O}(2)-\mathrm{U}(1)-\mathrm{O}(4) \# 1$ & $115.70(9)$ \\
$\mathrm{O}(1) \# 1-\mathrm{U}(1)-\mathrm{O}(2) \# 1$ & $91.58(11)$ & $\mathrm{O}(2) \# 1-\mathrm{U}(1)-\mathrm{O}(4) \# 1$ & $64.30(9)$ \\
$\mathrm{O}(1)-\mathrm{U}(1)-\mathrm{O}(2) \# 1$ & $88.42(11)$ & $\mathrm{O}(3) \# 1-\mathrm{U}(1)-\mathrm{O}(4) \# 1$ & $51.59(9)$ \\
$\mathrm{O}(2)-\mathrm{U}(1)-\mathrm{O}(2) \# 1$ & 180.0 & $\mathrm{O}(4)-\mathrm{U}(1)-\mathrm{O}(4) \# 1$ & 180.0 \\
$\mathrm{O}(1) \# 1-\mathrm{U}(1)-\mathrm{O}(3) \# 1$ & $91.48(12)$ & & \\
$\mathrm{O}(1)-\mathrm{U}(1)-\mathrm{O}(3) \# 1$ & $88.52(12)$ & \\
$\mathrm{O}(2)-\mathrm{U}(1)-\mathrm{O}(3) \# 1$ & $64.21(9)$ & & \\
$\mathrm{O}(2) \# 1-\mathrm{U}(1)-\mathrm{O}(3) \# 1$ & $115.79(9)$ & & \\
$\mathrm{O}(1) \# 1-\mathrm{U}(1)-\mathrm{O}(3)$ & $88.52(12)$ & \\
$\mathrm{O}(1)-\mathrm{U}(1)-\mathrm{O}(3)$ & $91.48(12)$ & \\
$\mathrm{O}(2)-\mathrm{U}(1)-\mathrm{O}(3)$ & $115.79(9)$ & \\
\end{tabular}


Table S2. Selected bond lengths $(\AA)$ and bond angles $\left({ }^{\circ}\right)$ for the uranyl centers in 2.

\begin{tabular}{|lc|lr|}
\hline $\mathrm{U}(1)-\mathrm{O}(1) \# 1$ & $1.7718(16)$ & $\mathrm{O}(4)-\mathrm{U}(1)-\mathrm{O}(4) \# 1$ & 180.0 \\
$\mathrm{U}(1)-\mathrm{O}(1)$ & $1.7718(16)$ & $\mathrm{O}(1) \# 1-\mathrm{U}(1)-\mathrm{O}(3) \# 1$ & $82.95(7)$ \\
$\mathrm{U}(1)-\mathrm{O}(2) \# 1$ & $2.4104(16)$ & $\mathrm{O}(1)-\mathrm{U}(1)-\mathrm{O}(3) \# 1$ & $97.05(7)$ \\
$\mathrm{U}(1)-\mathrm{O}(2)$ & $2.4104(16)$ & $\mathrm{O}(2) \# 1-\mathrm{U}(1)-\mathrm{O}(3) \# 1$ & $65.63(5)$ \\
$\mathrm{U}(1)-\mathrm{O}(4)$ & $2.4959(17)$ & $\mathrm{O}(2)-\mathrm{U}(1)-\mathrm{O}(3) \# 1$ & $114.37(5)$ \\
$\mathrm{U}(1)-\mathrm{O}(4) \# 1$ & $2.4959(17)$ & $\mathrm{O}(4)-\mathrm{U}(1)-\mathrm{O}(3) \# 1$ & $128.38(5)$ \\
$\mathrm{U}(1)-\mathrm{O}(3) \# 1$ & $2.5431(16)$ & $\mathrm{O}(4) \# 1-\mathrm{U}(1)-\mathrm{O}(3) \# 1$ & $51.62(5)$ \\
$\mathrm{U}(1)-\mathrm{O}(3)$ & $2.5431(16)$ & $\mathrm{O}(1) \# 1-\mathrm{U}(1)-\mathrm{O}(3)$ & $97.05(7)$ \\
$\mathrm{O}(1) \# 1-\mathrm{U}(1)-\mathrm{O}(1)$ & 180.0 & $\mathrm{O}(1)-\mathrm{U}(1)-\mathrm{O}(3)$ & $82.95(7)$ \\
$\mathrm{O}(1) \# 1-\mathrm{U}(1)-\mathrm{O}(2) \# 1$ & $96.15(7)$ & $\mathrm{O}(2) \# 1-\mathrm{U}(1)-\mathrm{O}(3)$ & $114.37(5)$ \\
$\mathrm{O}(1)-\mathrm{U}(1)-\mathrm{O}(2) \# 1$ & $83.84(7)$ & $\mathrm{O}(2)-\mathrm{U}(1)-\mathrm{O}(3)$ & $65.63(5)$ \\
$\mathrm{O}(1) \# 1-\mathrm{U}(1)-\mathrm{O}(2)$ & $83.84(7)$ & $\mathrm{O}(4)-\mathrm{U}(1)-\mathrm{O}(3)$ & $51.62(5)$ \\
$\mathrm{O}(1)-\mathrm{U}(1)-\mathrm{O}(2)$ & $96.16(7)$ & & \\
$\mathrm{O}(4) \# 1-\mathrm{U}(1)-\mathrm{O}(3)$ & $128.38(5)$ & & \\
$\mathrm{O}(3) \# 1-\mathrm{U}(1)-\mathrm{O}(3)$ & 180.0 & & \\
$\mathrm{O}(2) \# 1-\mathrm{U}(1)-\mathrm{O}(2)$ & 180.0 & \\
$\mathrm{O}(1) \# 1-\mathrm{U}(1)-\mathrm{O}(4)$ & $83.55(7)$ & & \\
$\mathrm{O}(1)-\mathrm{U}(1)-\mathrm{O}(4)$ & $96.44(7)$ & & \\
$\mathrm{O}(2) \# 1-\mathrm{U}(1)-\mathrm{O}(4)$ & $66.69(6)$ & & \\
$\mathrm{O}(2)-\mathrm{U}(1)-\mathrm{O}(4)$ & $113.31(6)$ & & \\
$\mathrm{O}(1) \# 1-\mathrm{U}(1)-\mathrm{O}(4) \# 1$ & $96.44(7)$ & \\
$\mathrm{O}(1)-\mathrm{U}(1)-\mathrm{O}(4) \# 1$ & $83.56(7)$ & \\
$\mathrm{O}(2) \# 1-\mathrm{U}(1)-\mathrm{O}(4) \# 1$ & $113.31(6)$ & \\
$\mathrm{O}(2)-\mathrm{U}(1)-\mathrm{O}(4) \# 1$ & $66.69(6)$ & \\
& & \\
\end{tabular}


Table S3. Selected bond lengths $(\AA)$ and bond angles $\left({ }^{\circ}\right)$ for the uranyl centers in $\mathbf{3}$.

\begin{tabular}{|lc|lc|}
\hline $\mathrm{U}(1)-\mathrm{O}(2)$ & $1.7683(19)$ & $\mathrm{O}(2)-\mathrm{U}(1)-\mathrm{O}(7)$ & $89.77(12)$ \\
$\mathrm{U}(1)-\mathrm{O}(1)$ & $1.7760(19)$ & $\mathrm{O}(1)-\mathrm{U}(1)-\mathrm{O}(7)$ & $89.85(12)$ \\
$\mathrm{U}(1)-\mathrm{O}(3)$ & $2.441(3)$ & $\mathrm{O}(3)-\mathrm{U}(1)-\mathrm{O}(7)$ & $172.67(9)$ \\
$\mathrm{U}(1)-\mathrm{O}(9)$ & $2.453(3)$ & $\mathrm{O}(9)-\mathrm{U}(1)-\mathrm{O}(7)$ & $118.70(9)$ \\
$\mathrm{U}(1)-\mathrm{O}(4)$ & $2.467(3)$ & $\mathrm{O}(4)-\mathrm{U}(1)-\mathrm{O}(7)$ & $121.99(9)$ \\
$\mathrm{U}(1)-\mathrm{O}(7)$ & $2.476(3)$ & $\mathrm{O}(2)-\mathrm{U}(1)-\mathrm{O}(8)$ & $95.27(15)$ \\
$\mathrm{U}(1)-\mathrm{O}(8)$ & $2.506(3)$ & $\mathrm{O}(1)-\mathrm{U}(1)-\mathrm{O}(8)$ & $85.26(15)$ \\
$\mathrm{U}(1)-\mathrm{O}(10)$ & $2.518(2)$ & $\mathrm{O}(3)-\mathrm{U}(1)-\mathrm{O}(8)$ & $121.70(9)$ \\
$\mathrm{O}(2)-\mathrm{U}(1)-\mathrm{O}(1)$ & $178.95(12)$ & $\mathrm{O}(9)-\mathrm{U}(1)-\mathrm{O}(8)$ & $168.55(9)$ \\
$\mathrm{O}(2)-\mathrm{U}(1)-\mathrm{O}(3)$ & $95.12(12)$ & $\mathrm{O}(7)-\mathrm{U}(1)-\mathrm{O}(1)-\mathrm{O}(8)$ & $70.11(9)$ \\
$\mathrm{O}(1)-\mathrm{U}(1)-\mathrm{O}(3)$ & $85.36(11)$ & $\mathrm{O}(2)-\mathrm{U}(1)-\mathrm{O}(10)$ & $52.19(8)$ \\
$\mathrm{O}(2)-\mathrm{U}(1)-\mathrm{O}(9)$ & $91.39(15)$ & $\mathrm{O}(1)-\mathrm{U}(1)-\mathrm{O}(10)$ & $92.21(10)$ \\
$\mathrm{O}(1)-\mathrm{U}(1)-\mathrm{O}(9)$ & $87.93(15)$ & $\mathrm{O}(3)-\mathrm{U}(1)-\mathrm{O}(10)$ & $86.74(10)$ \\
$\mathrm{O}(3)-\mathrm{U}(1)-\mathrm{O}(9)$ & $66.74(9)$ & $\mathrm{O}(9)-\mathrm{U}(1)-\mathrm{O}(10)$ & $118.76(9)$ \\
$\mathrm{O}(2)-\mathrm{U}(1)-\mathrm{O}(4)$ & O(4)-U(1)-O(10) & $52.34(9)$ \\
$\mathrm{O}(1)-\mathrm{U}(1)-\mathrm{O}(4)$ & $\mathrm{O}(7)-\mathrm{U}(1)-\mathrm{O}(10)$ & $171.39(9)$ \\
$\mathrm{O}(3)-\mathrm{U}(1)-\mathrm{O}(4)$ & $\mathrm{O}(8)-\mathrm{U}(1)-\mathrm{O}(10)$ & $66.37(9)$ \\
$\mathrm{O}(9)-\mathrm{U}(1)-\mathrm{O}(4)$ & $91.96(10)$ & & $117.93(8)$ \\
& $52.71(10)$ & & \\
\hline
\end{tabular}


Table S4. Selected bond lengths $(\AA)$ and bond angles $\left({ }^{\circ}\right)$ for the uranyl centers in 4.

\begin{tabular}{|c|c|c|c|}
\hline $\mathrm{U}(1)-\mathrm{O}(2)$ & $1.752(3)$ & $\mathrm{O}(5)-\mathrm{U}(1)-\mathrm{O}(3)$ & $114.40(8)$ \\
\hline $\mathrm{U}(1)-\mathrm{O}(1)$ & $1.757(3)$ & $\mathrm{O}(4)-\mathrm{U}(1)-\mathrm{O}(3)$ & $51.00(8)$ \\
\hline $\mathrm{U}(1)-\mathrm{O}(7)$ & $2.377(2)$ & $\mathrm{O}(2)-\mathrm{U}(1)-\mathrm{O}(6)$ & $91.62(14)$ \\
\hline $\mathrm{U}(1)-\mathrm{O}(5)$ & $2.473(2)$ & $\mathrm{O}(1)-\mathrm{U}(1)-\mathrm{O}(6)$ & $88.74(14)$ \\
\hline $\mathrm{U}(1)-\mathrm{O}(4)$ & $2.494(2)$ & $\mathrm{O}(7)-\mathrm{U}(1)-\mathrm{O}(6)$ & $176.16(11)$ \\
\hline $\mathrm{U}(1)-\mathrm{O}(3)$ & $2.519(2)$ & $\mathrm{O}(5)-\mathrm{U}(1)-\mathrm{O}(6)$ & $51.19(9)$ \\
\hline $\mathrm{U}(1)-\mathrm{O}(6)$ & $2.520(3)$ & $\mathrm{O}(4)-\mathrm{U}(1)-\mathrm{O}(6)$ & $114.23(8)$ \\
\hline $\mathrm{U}(1)-\mathrm{N}(7)$ & $2.668(3)$ & $\mathrm{O}(3)-\mathrm{U}(1)-\mathrm{O}(6)$ & $63.24(8)$ \\
\hline $\mathrm{O}(2)-\mathrm{U}(1)-\mathrm{O}(1)$ & $177.25(11)$ & $\mathrm{O}(2)-\mathrm{U}(1)-\mathrm{N}(7)$ & $89.33(12)$ \\
\hline $\mathrm{O}(2)-\mathrm{U}(1)-\mathrm{O}(7)$ & $92.07(13)$ & $\mathrm{O}(1)-\mathrm{U}(1)-\mathrm{N}(7)$ & $88.11(11)$ \\
\hline $\mathrm{O}(1)-\mathrm{U}(1)-\mathrm{O}(7)$ & 87.63(13) & $\mathrm{O}(7)-\mathrm{U}(1)-\mathrm{N}(7)$ & $62.19(8)$ \\
\hline $\mathrm{O}(2)-\mathrm{U}(1)-\mathrm{O}(5)$ & $91.14(14)$ & $\mathrm{O}(5)-\mathrm{U}(1)-\mathrm{N}(7)$ & $67.75(9)$ \\
\hline $\mathrm{O}(1)-\mathrm{U}(1)-\mathrm{O}(5)$ & $86.96(13)$ & $\mathrm{O}(4)-\mathrm{U}(1)-\mathrm{N}(7)$ & $126.82(8)$ \\
\hline $\mathrm{O}(7)-\mathrm{U}(1)-\mathrm{O}(5)$ & $129.78(9)$ & $\mathrm{O}(3)-\mathrm{U}(1)-\mathrm{N}(7)$ & 177.21(8) \\
\hline $\mathrm{O}(2)-\mathrm{U}(1)-\mathrm{O}(4)$ & $88.15(12)$ & $\mathrm{O}(6)-\mathrm{U}(1)-\mathrm{N}(7)$ & $118.93(8)$ \\
\hline $\mathrm{O}(1)-\mathrm{U}(1)-\mathrm{O}(4)$ & $94.19(12)$ & & \\
\hline $\mathrm{O}(7)-\mathrm{U}(1)-\mathrm{O}(4)$ & $64.83(8)$ & & \\
\hline $\mathrm{O}(5)-\mathrm{U}(1)-\mathrm{O}(4)$ & $165.39(9)$ & & \\
\hline $\mathrm{O}(2)-\mathrm{U}(1)-\mathrm{O}(3)$ & $88.85(13)$ & & \\
\hline $\mathrm{O}(1)-\mathrm{U}(1)-\mathrm{O}(3)$ & $93.74(12)$ & & \\
\hline $\mathrm{O}(7)-\mathrm{U}(1)-\mathrm{O}(3)$ & $115.77(8)$ & & \\
\hline
\end{tabular}


Table S5. Bond valence summations for compound $\mathbf{1 .}$

\begin{tabular}{|c|c|c|}
\hline $\begin{array}{c}\text { Bond valence parameter for } \\
\text { U(VI) and }{ }^{1}\end{array}$ & 2.051 & \\
\hline Bonded atom to U1 & U-O Bond distance $(\AA)$ & $\begin{array}{c}\text { Bond valence summation } \\
(\mathrm{vu})\end{array}$ \\
\hline O1 & 1.762 & 1.745 \\
\hline O1' & 1.762 & 1.745 \\
\hline O2 & 2.418 & 0.493 \\
\hline O2' & 2.418 & 0.493 \\
\hline O3 & 2.500 & 0.421 \\
\hline O3' & 2.500 & 0.421 \\
\hline O4 & 2.516 & 0.408 \\
\hline O4' & 2.516 & 0.408 \\
\hline Overall charge on U(VI) & & 6.134 \\
\hline
\end{tabular}

Bond valence summations for oxygen atoms in 1. The values for terminal oxygen atoms $\mathrm{O} 2$ and $\mathrm{O} 2$ ' indicate that both atoms are water molecules. ${ }^{1-3}$

Table S6. Bond valence summations for compound 2.

\begin{tabular}{|c|c|c|}
\hline $\begin{array}{l}\text { Bond valence parameter for } \\
\text { U(VI) and }{ }^{1}\end{array}$ & 2.051 & \\
\hline Bonded atom to U1 & U-O Bond distance $(\AA)$ & $\begin{array}{c}\text { Bond valence summation } \\
(\mathrm{vu})\end{array}$ \\
\hline O1 & 1.772 & 1.715 \\
\hline O1' & 1.772 & 1.715 \\
\hline O2 & 2.41 & 0.500 \\
\hline O2' & 2.41 & 0.500 \\
\hline O3 & 2.543 & 0.387 \\
\hline O3' & 2.543 & 0.387 \\
\hline O4 & 2.496 & 0.424 \\
\hline O4' & 2.496 & 0.424 \\
\hline Overall charge on U(VI) & & 6.055 \\
\hline
\end{tabular}

Bond valence summations for oxygen atoms in 2 . The values for terminal oxygen atoms $\mathrm{O} 2$ and $\mathrm{O} 2$ ' indicate that both atoms are water molecules. ${ }^{1-3}$ 


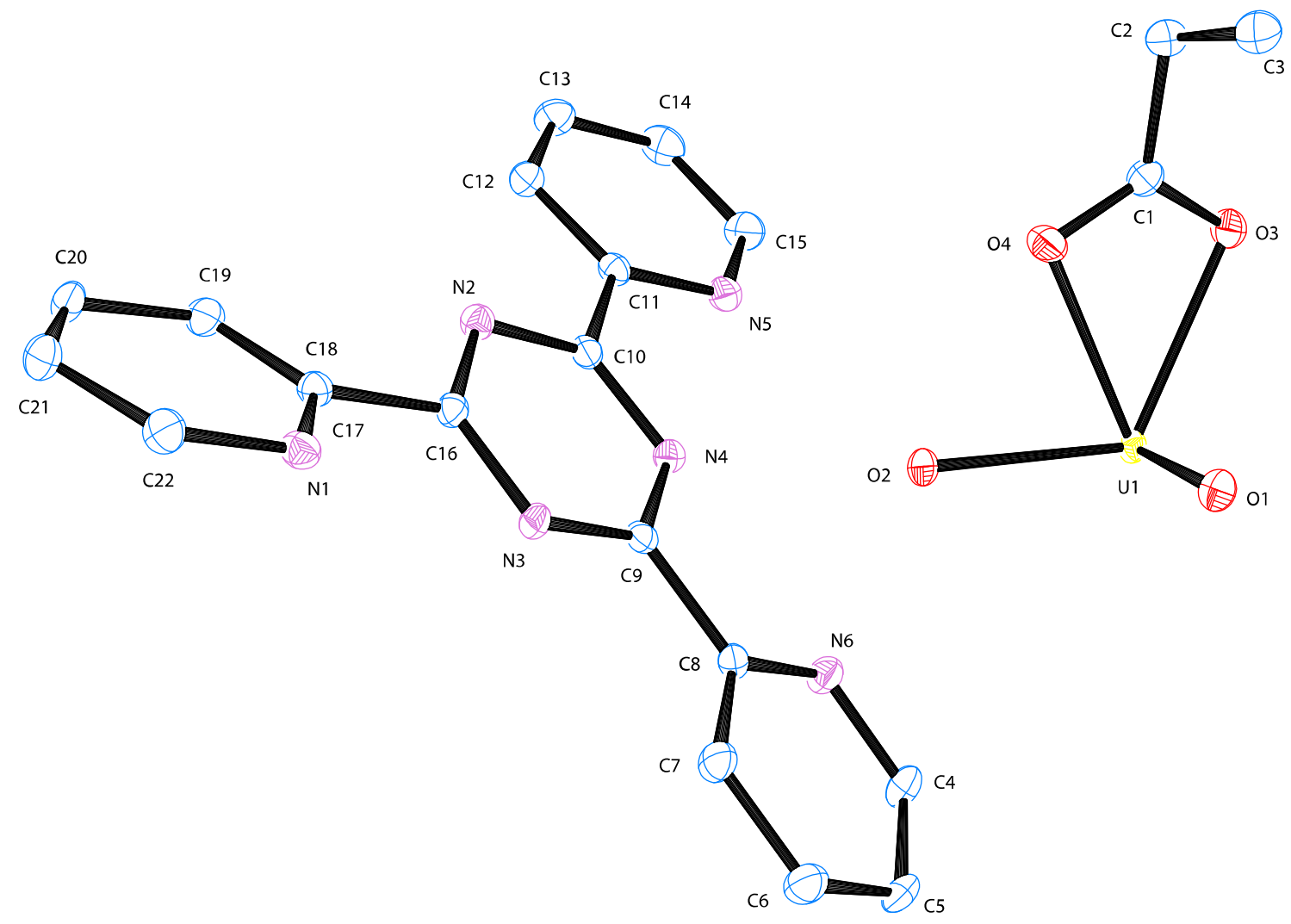

Figure S1. ORTEP representation of 1. Thermal ellipsoids are shown at 50\% probability. Hydrogen atoms have been omitted. 


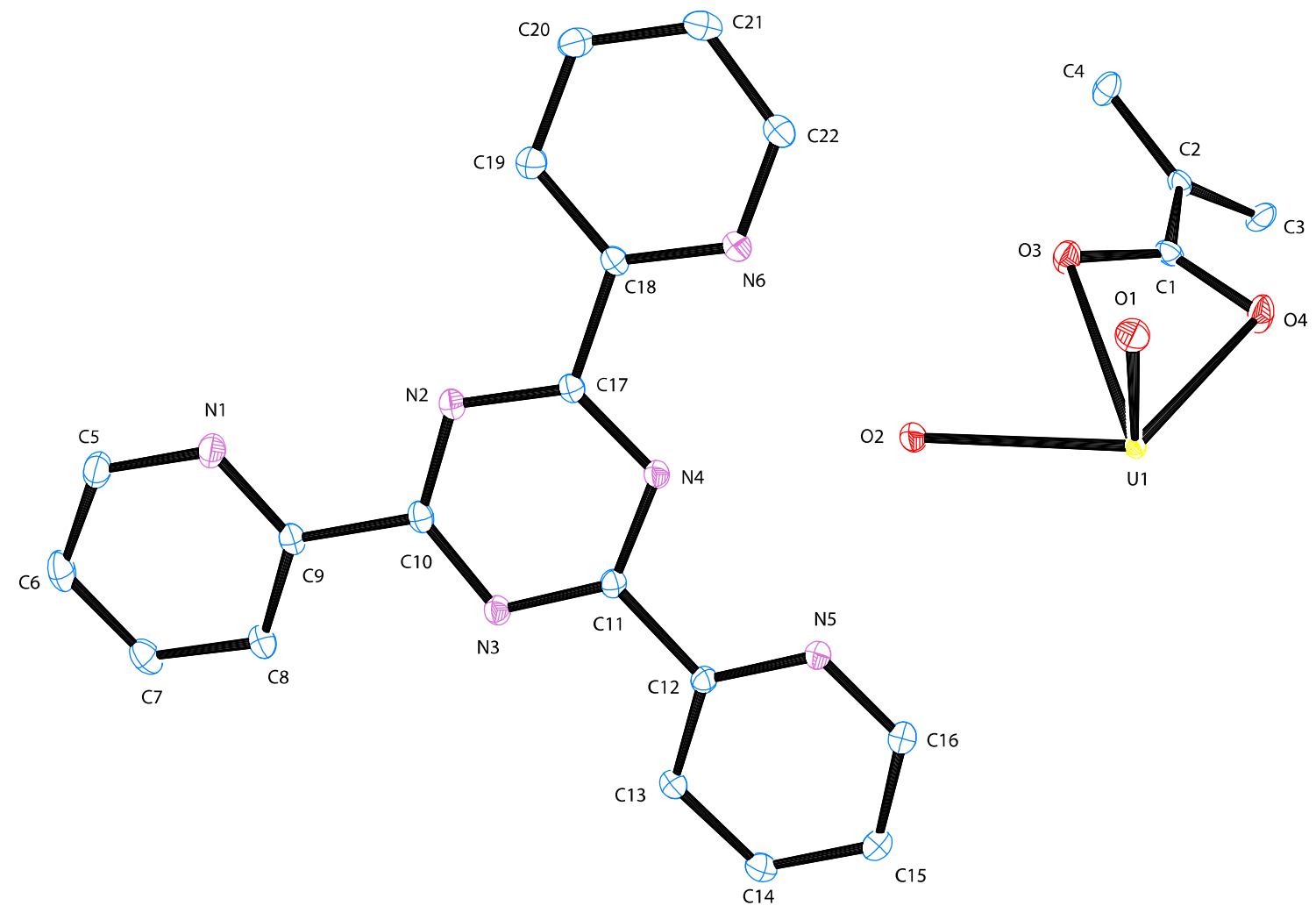

Figure S2. ORTEP representation of 2. Thermal ellipsoids are shown at $50 \%$ probability. Hydrogen atoms have been omitted. 


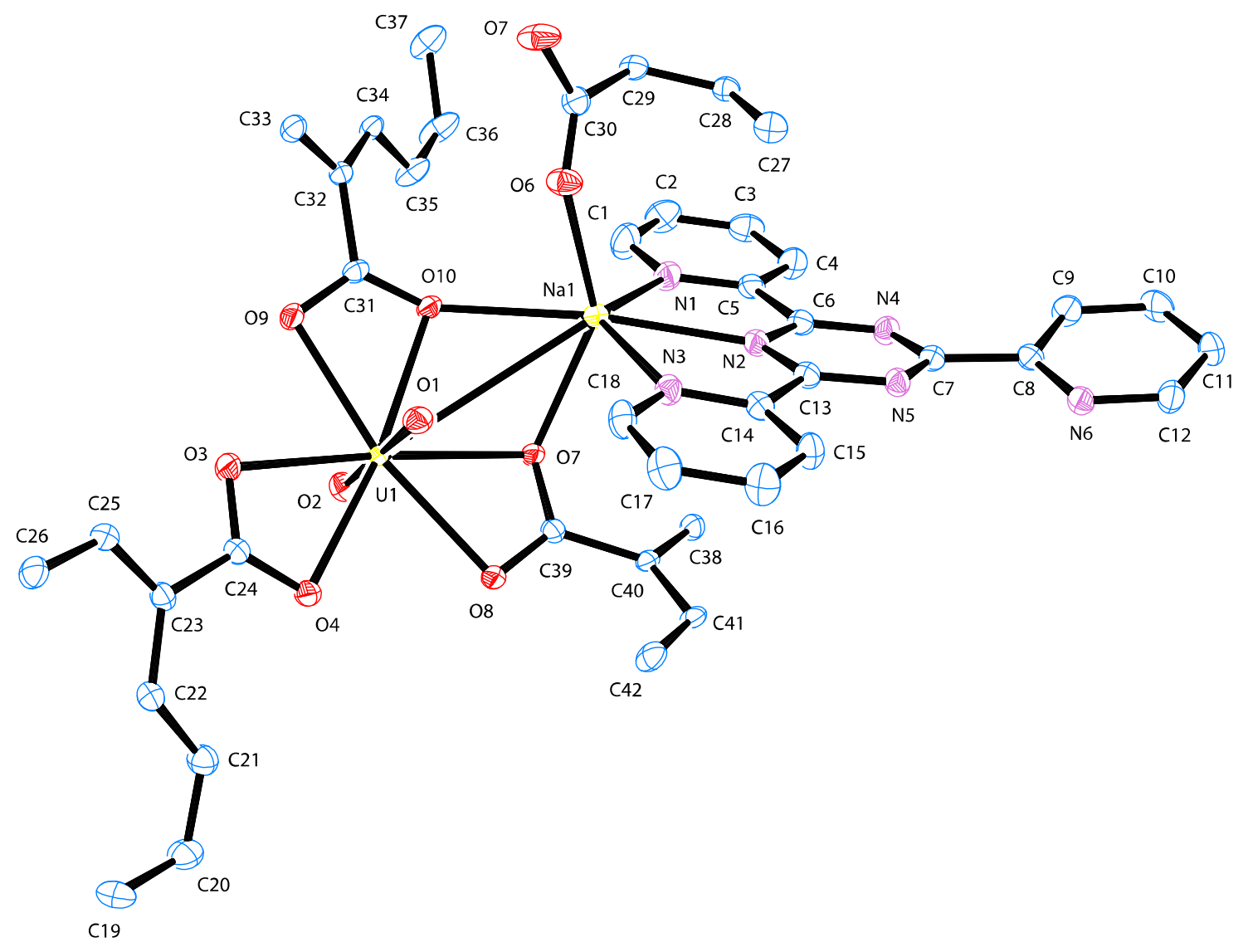

Figure S3. ORTEP representation of 3. Thermal ellipsoids are shown at 50\% probability. Hydrogen atoms and solvent water Ow1 have been omitted. 


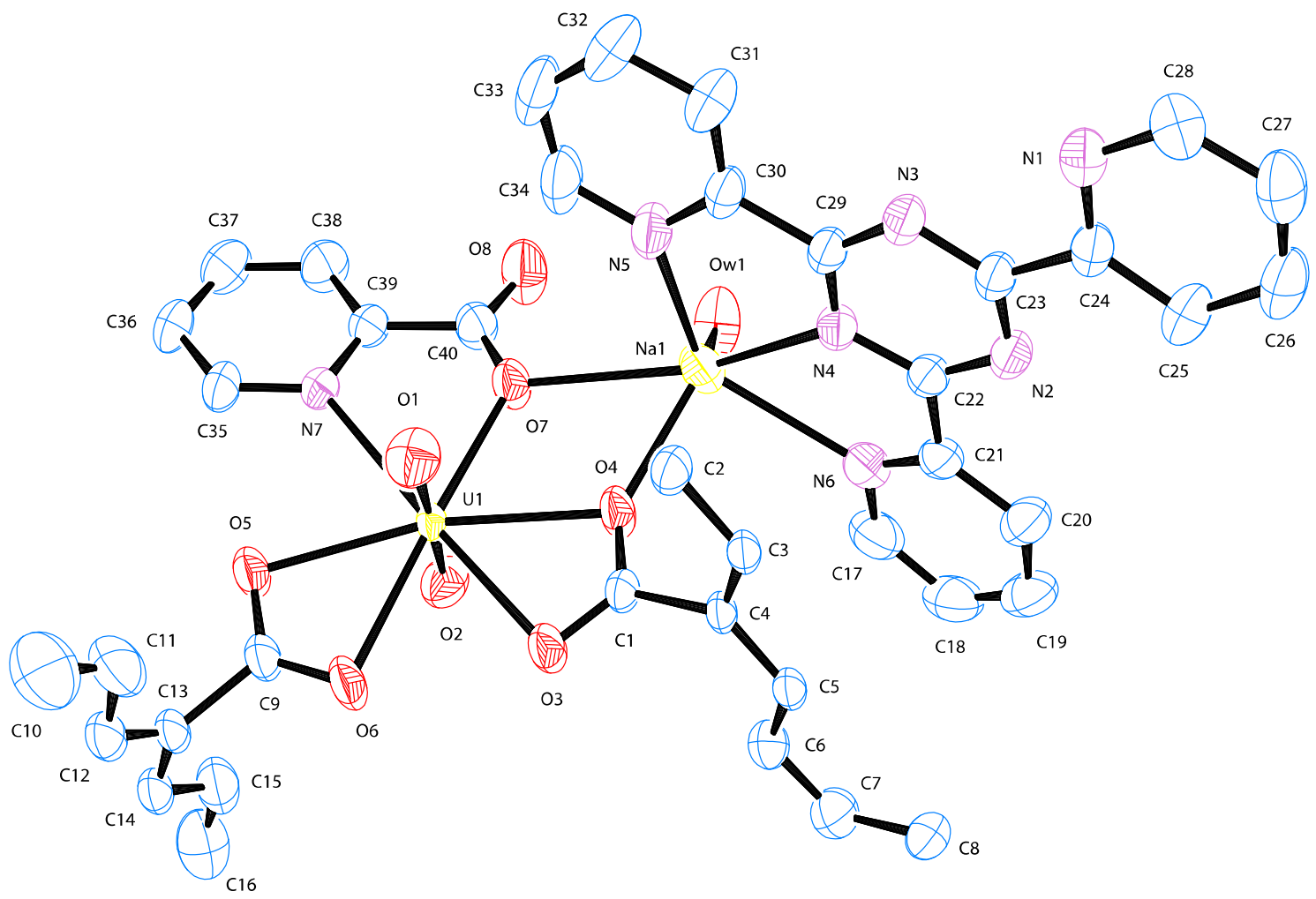

Figure S4. ORTEP representation of 4. Thermal ellipsoids are shown at 50\% probability. Hydrogen atoms have been omitted. 


\section{Compound 1}

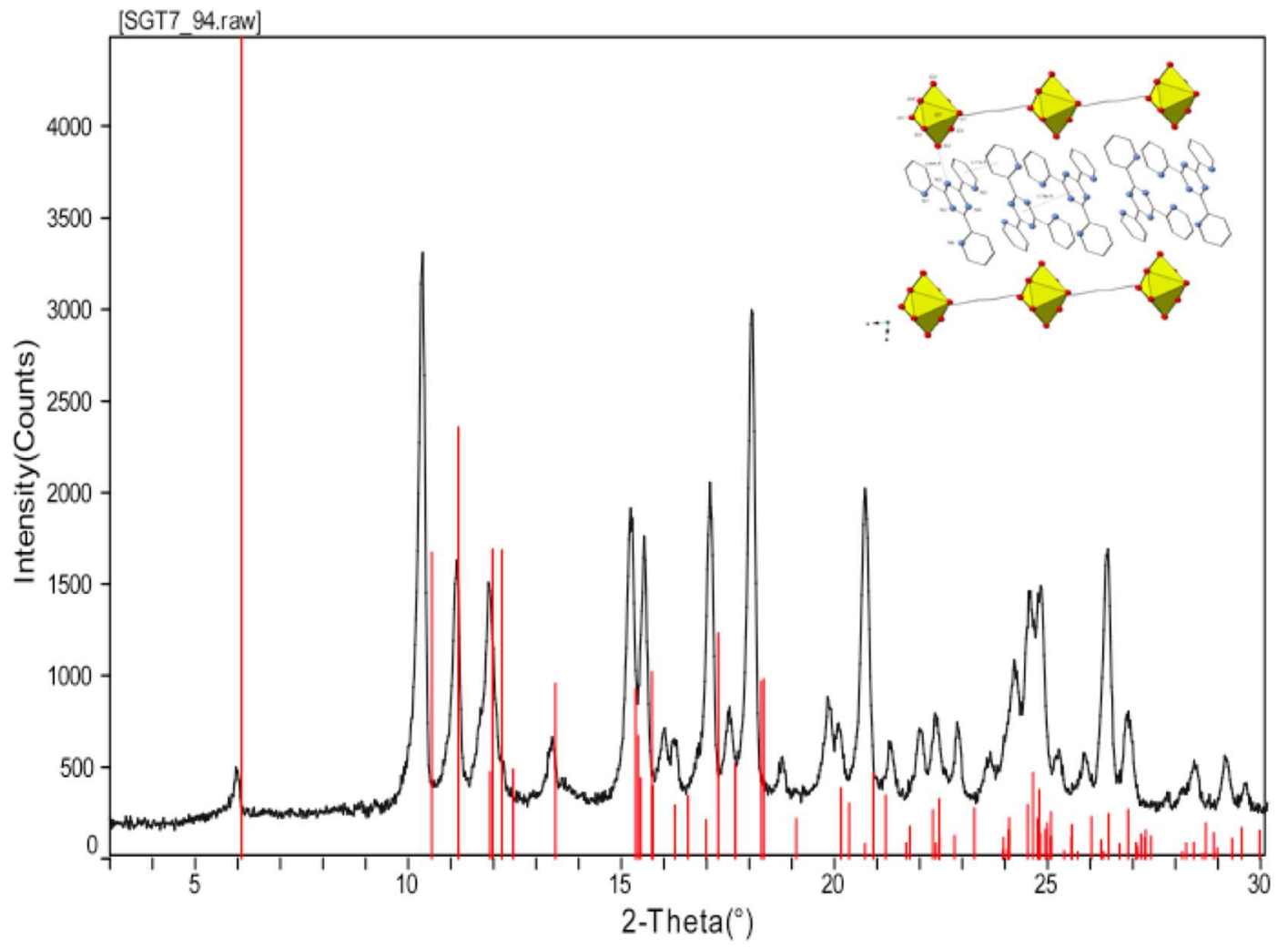

Figure S5. Observed and calculated powder X-ray diffraction pattern of $\mathbf{1}$. 


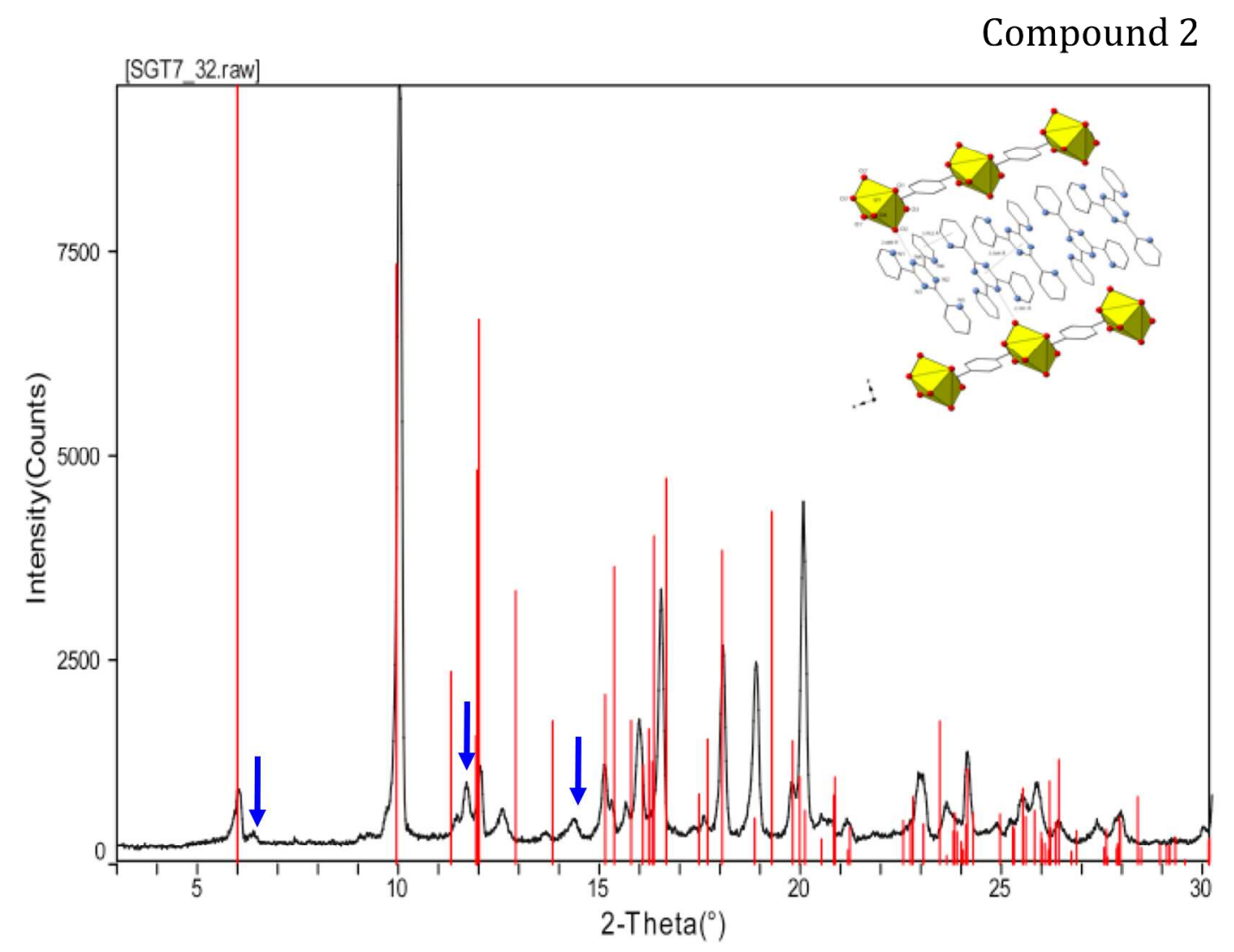

Figure S6. Observed and calculated powder X-ray diffraction pattern of 2. Impurities present in the bulk sample are indicated by blue arrows. 


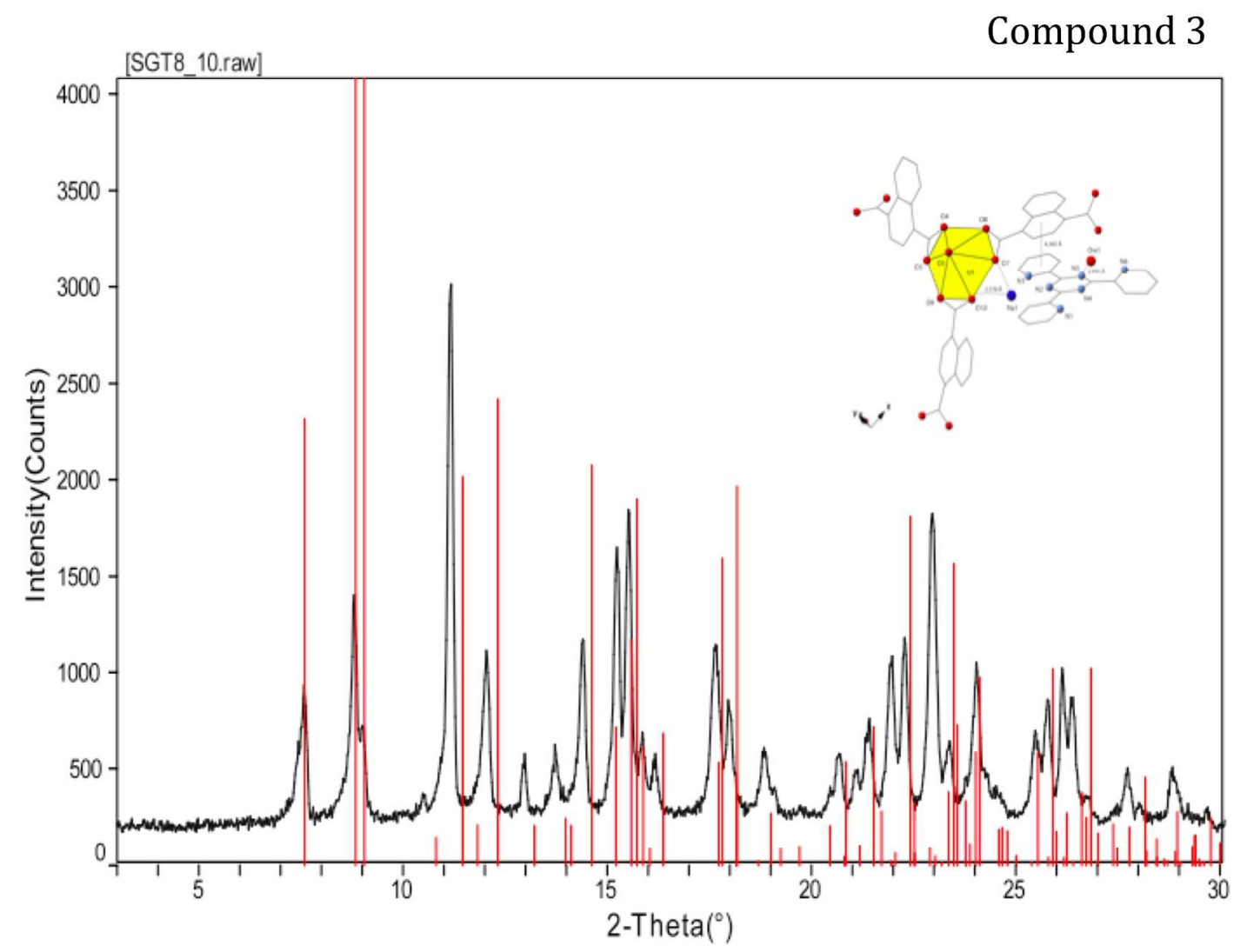

Figure S7. Observed and calculated powder X-ray diffraction pattern of $\mathbf{3}$. 


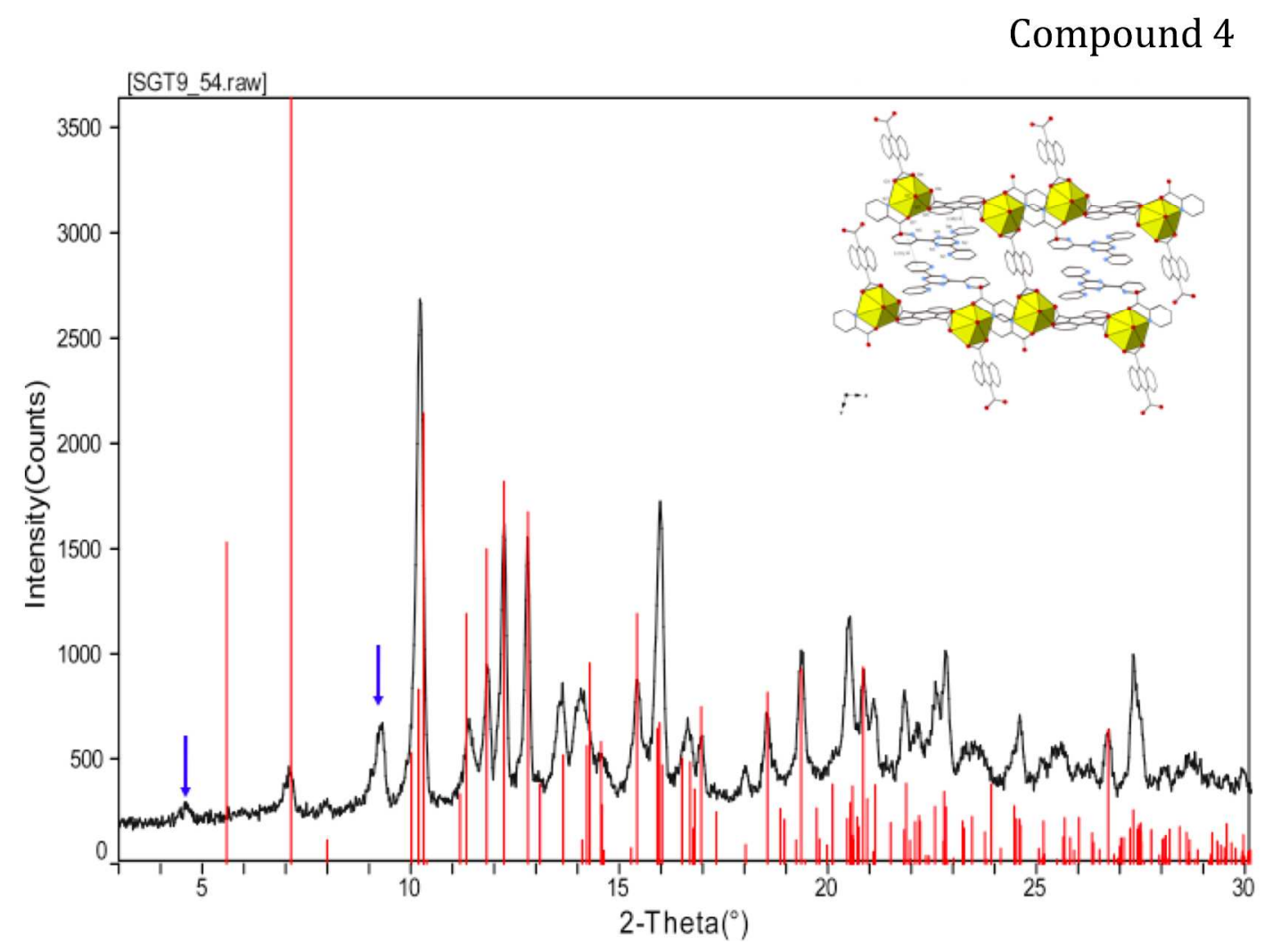

Figure S8. Observed and calculated powder X-ray diffraction pattern of 4. Impurities present in the bulk sample are indicated by blue arrows. 


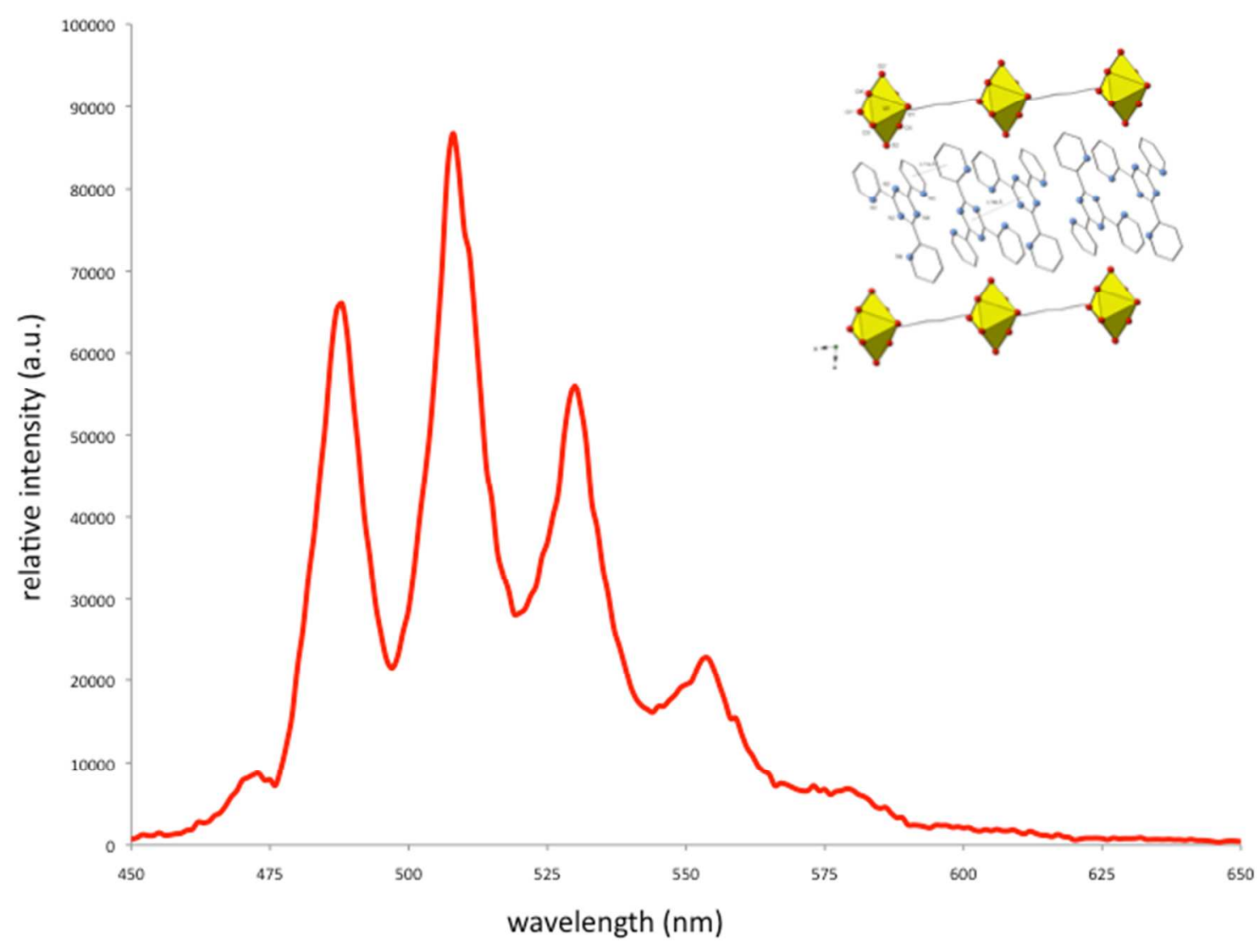

Figure S9. Room temperature solid state emission spectrum of $\mathbf{1}\left(\lambda_{\mathrm{ex}}=420 \mathrm{~nm}\right)$. 


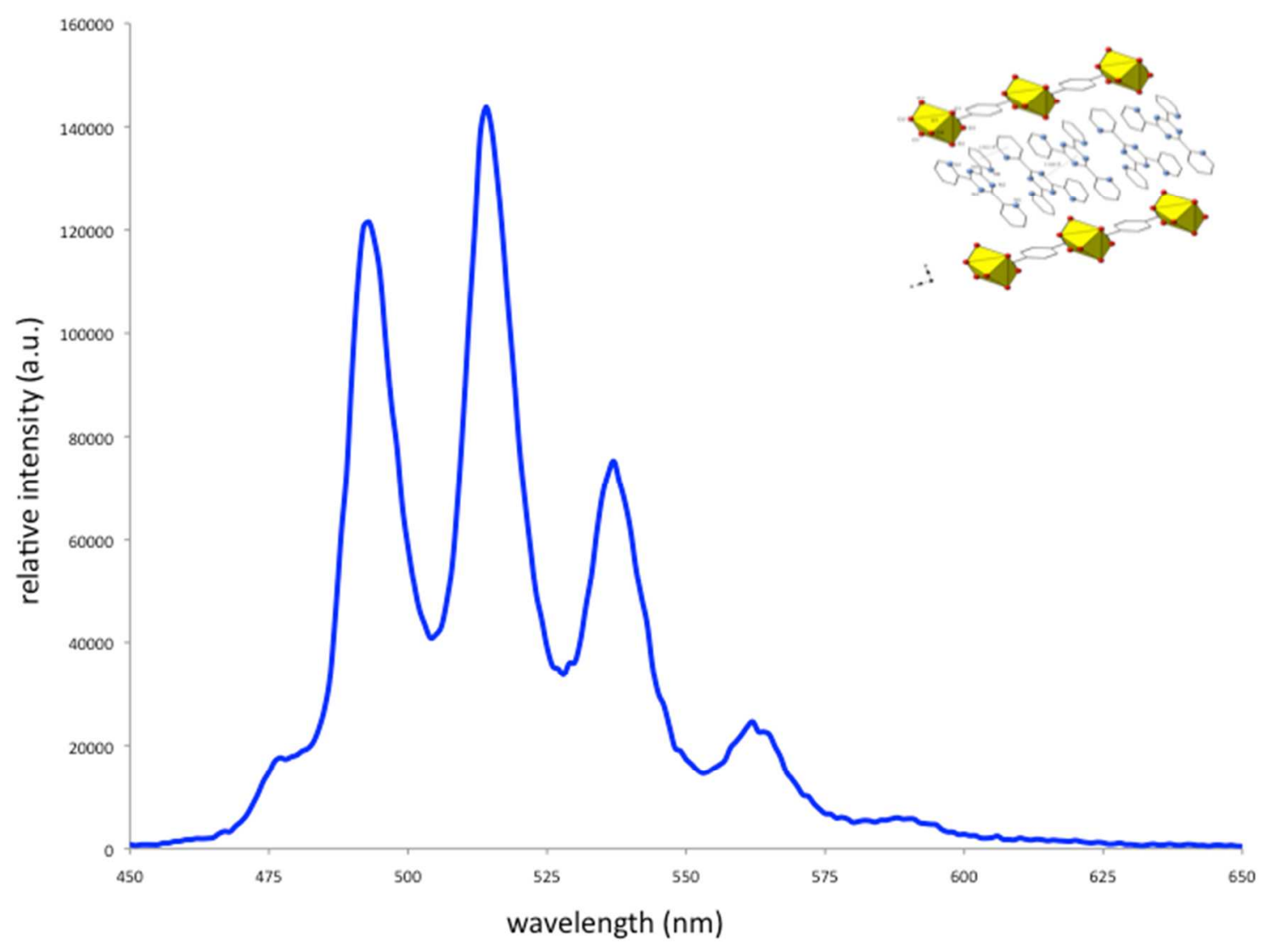

Figure S10. Room temperature solid state emission spectrum of $2\left(\lambda_{\mathrm{ex}}=420 \mathrm{~nm}\right)$. 


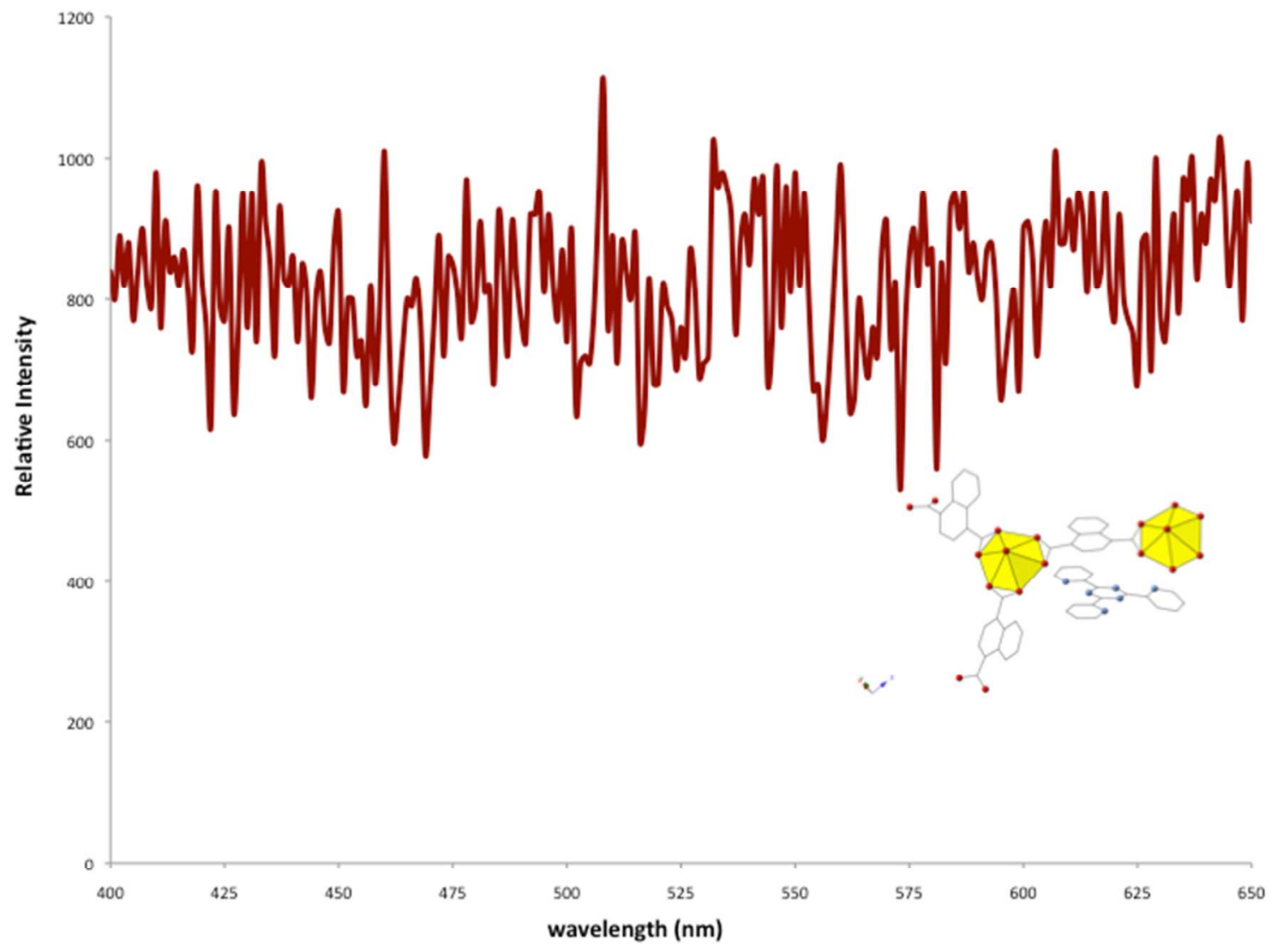

Figure S11. Room temperature solid state emission spectrum of $\mathbf{3}\left(\lambda_{\mathrm{ex}}=365 \mathrm{~nm}\right)$. 


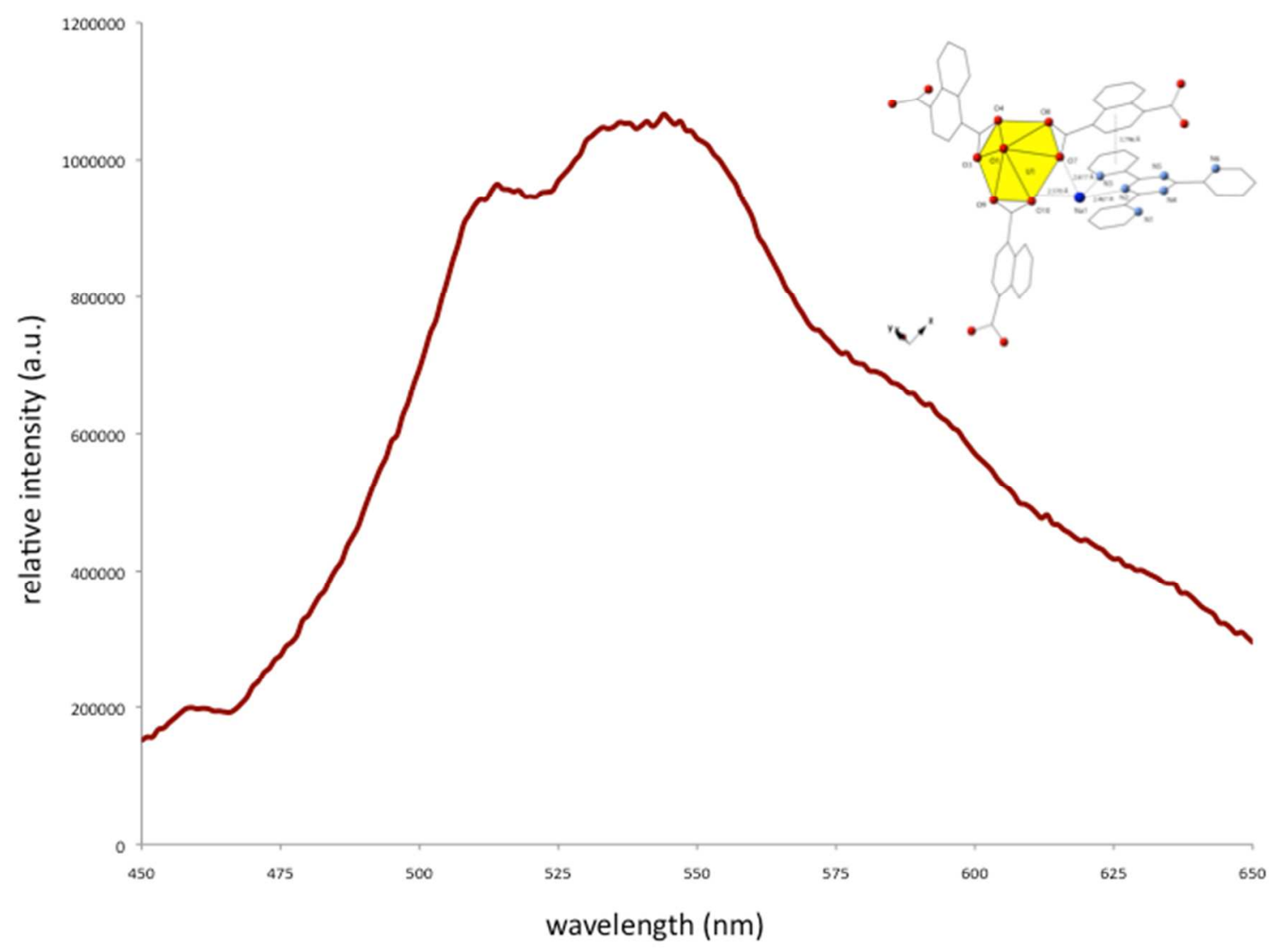

Figure S12. Room temperature solid state emission spectrum of $\mathbf{3}\left(\lambda_{\mathrm{ex}}=420 \mathrm{~nm}\right)$. 


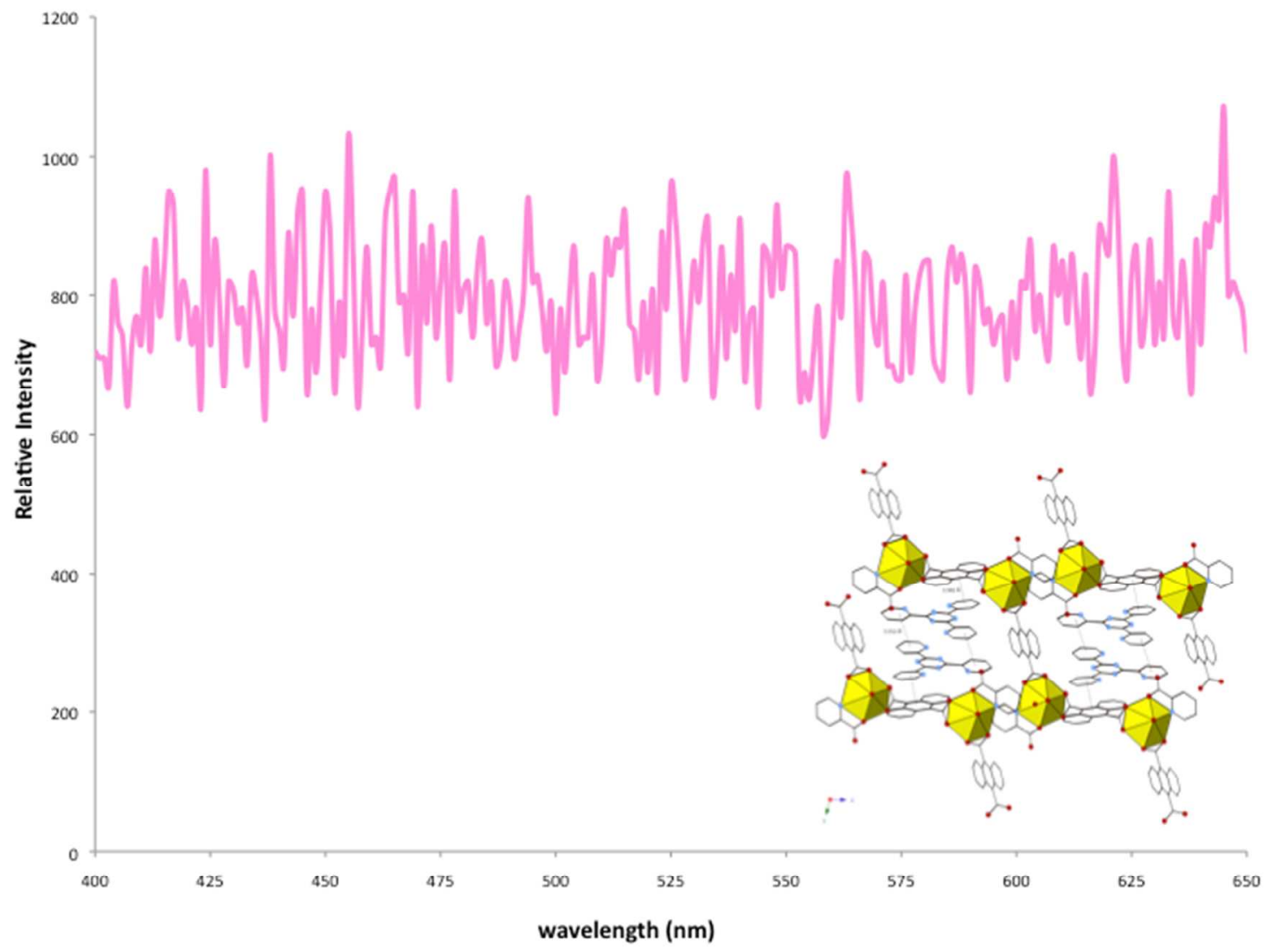

Figure S13. Room temperature solid state emission spectrum of $4\left(\lambda_{\mathrm{ex}}=365 \mathrm{~nm}\right)$. 


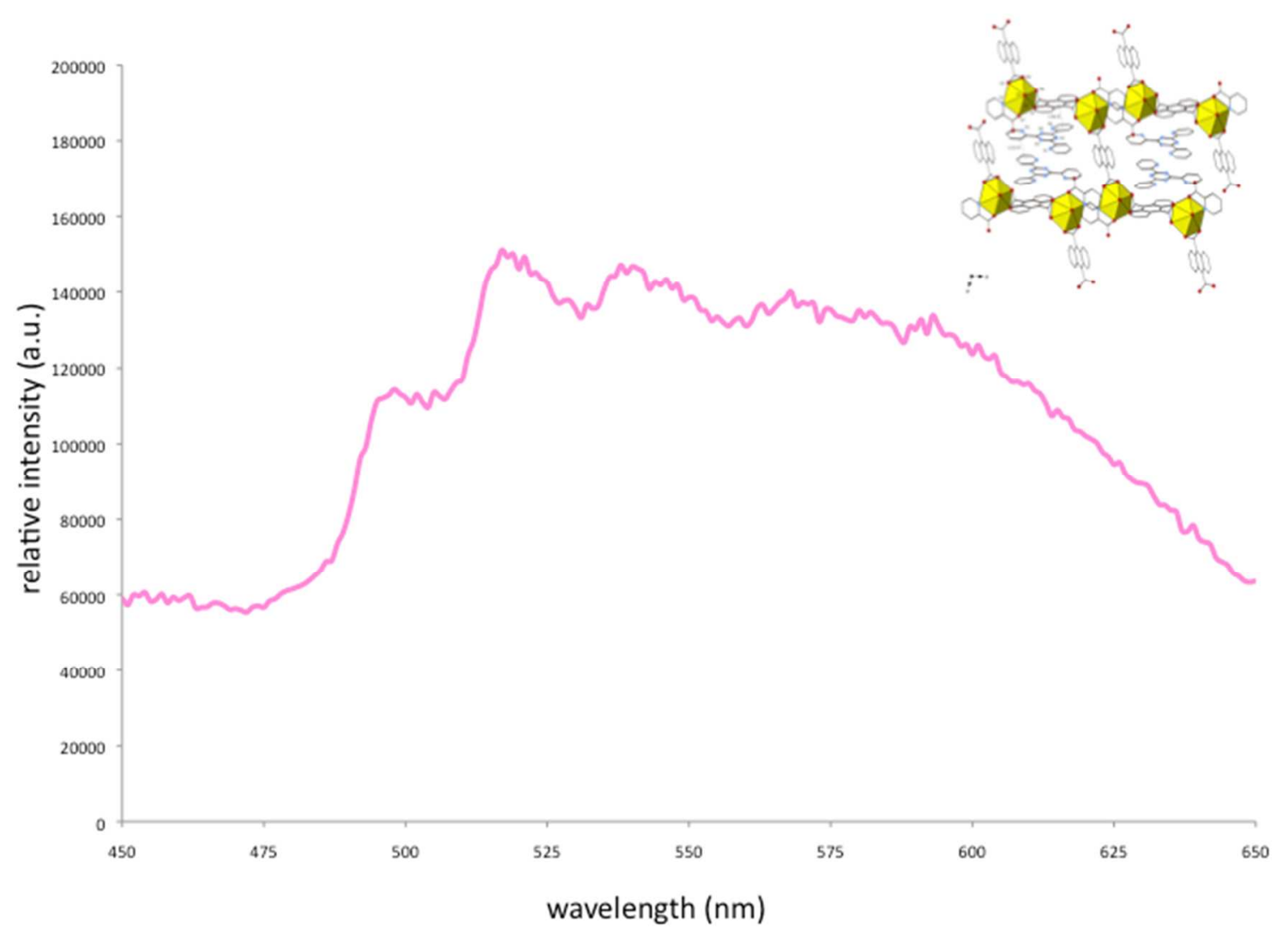

Figure S14. Room temperature solid state emission spectrum of $4\left(\lambda_{\mathrm{ex}}=420 \mathrm{~nm}\right)$.

General Determination of Emission Shift. To determine the emission shift of $\mathbf{1}$ and 2, the peak positions of each spectrum were measured. The peak positions of 1 were superimposed to the emission peak positions of $\mathbf{2}$ and then the energy difference between each peak was calculated. A summary of these results can be found in Table S1.

Table S1. Summary of emission peak positions and shifts of 1 and $\mathbf{2}$ (excitation: 365 $\mathrm{nm})$.

\begin{tabular}{ccccccc}
\hline Compound & $\begin{array}{c}\text { Position 1 } \\
\left(\mathrm{cm}^{-1}\right)\end{array}$ & $\begin{array}{c}\text { Position 2 } \\
\left(\mathrm{cm}^{-1}\right)\end{array}$ & $\begin{array}{c}\text { Position 3 } \\
\left(\left(\mathrm{cm}^{-1}\right)\right.\end{array}$ & $\begin{array}{c}\text { Position 4 } \\
\left(\mathrm{cm}^{-1}\right)\end{array}$ & $\begin{array}{c}\text { Position 5 } \\
\left(\mathrm{cm}^{-1}\right)\end{array}$ & $\begin{array}{c}\text { Average } \\
\left(\mathrm{cm}^{-1}\right)\end{array}$ \\
\hline $\mathbf{1}$ & 20,534 & 19,724 & 18,904 & 18,083 & 17,271 & 18,903 \\
$\mathbf{2}$ & 20,325 & 19,493 & 18,657 & 17,794 & 17,036 & 18,661 \\
Energy shift & 209 & 231 & 257 & 289 & 235 & 242 \\
& & & & & & \\
\hline
\end{tabular}




\section{References}

(1) Burns, P. C. Can. Mineral. 1997, 35, 1551-1570.

(2) Burns, P. C. Can. Mineral. 2005, 43, 1839-1894.

(3) Giesting, P. A.; Burns, P. C. Crystallogr. Rev. 2006, 2006, 205-255. 